Research Article

\title{
Learning from error: identification and analysis of causative factors leading to medication error in an inpatient hospital setting
}

\author{
Subodh Kumar ${ }^{1}$, Anju Madhwar ${ }^{2}$, Anuj Kumar Pathak ${ }^{3}$, Dibyajyoti Saikia ${ }^{4}$
}

\begin{abstract}
${ }^{1}$ Department of Pharmacology, BRD Medical College, Gorakhpur, Uttar Pradesh, India ${ }^{2}$ Department of Pharmacology, SRMSIMS, Bareilly, Uttar Pradesh, India

${ }^{3}$ Department of Pharmacology, IGIMS, Patna, Bihar, India ${ }^{4}$ Department of Pharmacology, AIIMs, New-Delhi, India
\end{abstract}

Received: 29 March 2016 Accepted: 07 May 2016

*Correspondence to: Dr. Subodh Kumar, Email: drsubodh27@gmail.com

Copyright: (C) the author(s), publisher and licensee Medip Academy. This is an openaccess article distributed under the terms of the Creative Commons Attribution NonCommercial License, which permits unrestricted noncommercial use, distribution, and reproduction in any medium, provided the original work is properly cited.

\begin{abstract}
Background: Medication errors are preventable causes of medical error. These errors may happen at various steps of medication process. This study tries to find common errors during four stages i.e. prescribing, transcribing, dispensing and administration.

Methods: It was a cross sectional study in a tertiary care teaching hospital in north India. Five hundred patients were randomly selected for the study. Medication error was checked at different level by a medication audit tool. Direct observation, chart reviews and personal communication with patients, nurses and pharmacist were done to complete the details of tool. Root cause analysis was done after discussing with concerned professionals. Severity of the medication errors were assessed using national coordinating council for medication error reporting and prevention (NCCMERP) proposed medication error index.

Results: Medication error rate was found to be around $50 \%$. i.e. every one of two patients received some form of error. Prescribing error were most common cause of error followed by administration error and transcription error. Dispensing error was least common. Root cause analysis indicated casual attitude, inadequate knowledge and training as the main causes.

Conclusions: This study was an initial step in recognising error prone areas of medication management. It can be used to develop standard procedures and formulating guidelines for prevention of such errors.
\end{abstract}

Keywords: Medication error, Prescribing error, Administration error, Transcription error, Dispensing error

\section{INTRODUCTION}

Human error in medical practice can occur at various levels such as diagnosis, medication, clerical and treatment procedures. ${ }^{1}$ Medication errors comprise common causes of these errors. National coordinating council for medication error reporting and prevention (NCCMERP) defines medication error as 'any preventable event that may cause or lead to inappropriate medication use or patient's harm while the medication is in the control of the health care professional, patient, or consumer. ${ }^{2}$ These errors may result in therapeutic failure and adverse drug reactions as well as wasting of resources. A landmark report by institute of medicine (IOM) in 2000, To Err Is human: building a safer health care system, found that medication errors were important cause of preventable deaths amounting to around 44,000 to 98,000 preventable deaths each year, with an associated cost of $\$ 17$ to $\$ 29$ billion in US hospitals. ${ }^{3}$ In one study published in JAMA in the inpatients of two teaching hospitals, it was reported that $30 \%$ of patient injuries occurring in a teaching hospital resulted from preventable adverse drug effects. Estimated excess hospital costs attributable per adverse drug event were 
$\$ 4700$ in a year. ${ }^{4}$ In India there is no system of reporting of medication errors. Few private set ups routinely does medication error detection and reporting as part of internal assessment or as requirement for various accreditation process. But generally such a system is lacking in private and government institutions. Most of the medication error remains unreported in health care settings. It is noticed only when there is occurrence of some sentinel event which raises eyebrows. There is also general tendency of covering up of such incidents fearing penalization. This is due to unawareness and lack of regulatory policies among health care professionals. Hence there is lack of data on medication error in India.

A questionnaire based survey to assess the knowledge regarding medication errors among health care professionals done across India has concluded that $27.5 \%$ of the respondents had poor knowledge. ${ }^{5}$ Various methods for detection of medication errors have been described. These include chart review, incident reporting, direct observation and from administrative database. ${ }^{6-8}$ Research generally combines one or more methods. The study aims to promote safety in medication use and ensure quality in the healthcare service by effective utilization of a clinical pharmacologist. The study objective was to detect, categorize and analyse medication errors to ensure rational drug use in the hospital.

\section{METHODS}

It was a descriptive cross sectional study on five hundred hospitalized patients in a tertiary care teaching hospital in north India. The study was conducted from March to June 2015. Opportunities of error at various level of medication process were identified and classified (Figure 1). Medication errors were detected by direct observation and chart review method. In order to prevent medication errors and reduce the risks of harm, organizations need tools to detect them. A 'medication error audit tool' to detect medication error was developed to evaluate types and incidence of medication errors in four stages including prescribing, transcribing, dispensing and administering using prevalent studies and guidelines. ${ }^{9-14}$ The components of the tool is given in (Table 1). It was validated in ten patients. The tool was subsequently modified and used for detection of medication error. Complete details of medication error audit tool was filled by reviewing prescriptions, medication charts, nursing records, medicine order sheet, dispensed medicine and personal interviews with physicians, nurses, pharmacist and patients. The available information was noted and cross checked. Collected details were then evaluated to detect the prescribing, transcription, administration and dispensing error. The institutional ethics committee (IEC-SRMSIMS) approval was taken prior to the study. Permission to access patient related data were obtained from medical superintendent. Confidentiality of patients was maintained according to ethical principles of world medical association declaration of Helsinki.

Table 1: Component of medication errors in medical audit tool.

\begin{tabular}{|llll|}
\hline Prescribing error & Transcription error & Dispensing error & Administration error \\
\hline Missed dose/dosage & Missed dose/dosage & Wrong drug & Overdose/under dose \\
\hline form/unit/frequency & form/unit & Wrong dose & Wrong route \\
\hline Route/dilution & Transcription not legible & Wrong dosage form & Wrong frequency \\
\hline Prescription not capital/legible & Wrong dose & & Wrong dilution \\
\hline Unstandard abbreviation & Wrong dosage form & & Wrong time/missed drug \\
\hline Wrong dose & Wrong unit & Wrong drug \\
\hline Wrong dosage form & & Mixing two or more drugs \\
\hline Wrong unit & & \\
\hline Wrong frequency & & \\
\hline Wrong route & & \\
\hline Wrong dilution & & \\
\hline Therapeutic duplication & & \\
\hline Drug interaction & & \\
\hline
\end{tabular}

As given in (Table 1) the components of prescribing error included errors in dose, dosage form, unit, frequency, route, dilution, therapeutic duplication, drug interaction and illegible prescription. It was checked by analysing prescriptions. Drug interaction was checked by drug interaction checker available online. ${ }^{15}$ Only moderate and severe interactions were taken for consideration.

Transcription error was defined as error in copying of prescription. It may be during copying to another paper, 
computer, facsimile or medication administration record. ${ }^{16}$ In our setting, once the order is written, the nurse records the medications ordered onto a medication list (medication sheet). One of the nurses then transcribes the medication orders from the medication sheet onto a request list, which is sent to the pharmacy department. Transcription errors were checked in medication order and request sheet by comparing drug list written by staff nurse to prescription order. It included drug name, dose, dosage form, units and other general information such as patient name, registration number, date and signature. Dispensing error was checked by comparing drugs dispensed by pharmacist to the medicine sheet. Administration error was defined as deviation from the prescriber's medication order as written on the patient's chart, manufacturers' instructions or relevant institutional policies. Administration error was observed while nurses prepared and administered drugs to the patients. It included error in giving drug, doses (under or over dose), route frequency, dilution, mixing drugs in same syringe and time of administering the drug. Medication errors were also assessed for its severity level by using the national coordination council for medication error reporting prevention proposed medication error index.

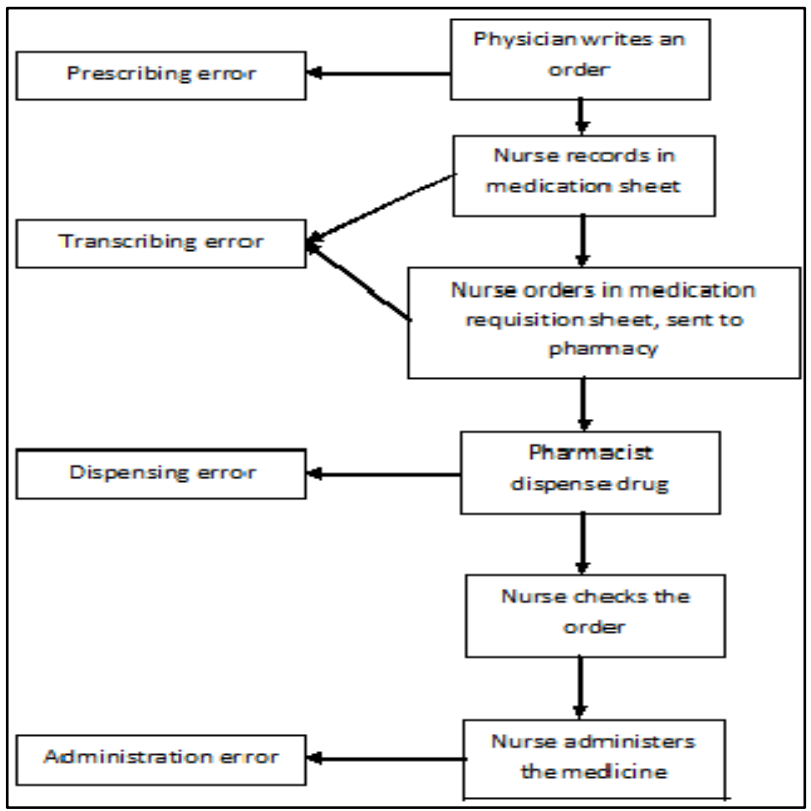

Figure 1: Medication process and level of detection of errors.

Analysis of what may have led to error was done after discussing with involved professionals. It is called root cause analysis (RCA). ${ }^{17}$ It basically requires answering three questions 1) What was the error? 2) Why the error? 3) What could be done to prevent it? The joint commission on accreditation of healthcare organizations requires root cause analyses and action plan for all sentinel events. A sentinel event is any unexpected occurrence involving death or serious physical or psychological injury, or the risk thereof. Serious injuries specifically include a loss of limb or function. It has given a root cause analyses and action plan framework template. $^{18}$

\section{Categorization of error as per NCC MERP Index}

National coordinating council for medication error reporting and prevention (NCCMERP) proposed medication error index was used to assess the severity of medication error. ${ }^{19}$ It classifies an error according to the severity of the outcome. The index considers factors such as whether the error reached the patient and, if the patient was harmed, to what degree the harm was.

There was no previous similar study in our institute which could have been used for calculation of sample size. We evaluated total 10 wards with approximately 50 prescription from each ward making it 500. Further, Similar works in past has been done with mentioned sample size. $^{20,21}$

\section{RESULTS}

Among 500 cases of the patients followed during the study period $357(71.4 \%)$ were male and $143(28.6 \%)$ were females. A total of 293 cases of medication errors were detected. Several prescriptions and transcriptions were having more than one error. As shown in (Table 2). Prescribing errors form the majority of the error followed by administration, transcription and dispensing error. If we count all errors in a prescription or transcription, the total number of medication error was 252 as given in (Table 3).

Table 2: Frequency and percentage of errors.

\begin{tabular}{|lcc|}
\hline Medication error & No. of errors & Percentage \\
\hline Prescribing error & 110 & 37.54 \\
\hline Transcription error & 68 & 23.21 \\
\hline Dispensing error & 13 & 4.44 \\
\hline Administration error & 102 & 34.81 \\
\hline
\end{tabular}

*Percentage has been calculated from total error which was 293.

Table 3: Frequency and percentage of medication error for all the errors in a prescription or transcription has been counted as one.

\begin{tabular}{|lcc|}
\hline Medication error & No. of error & "Percentage \\
\hline Prescribing error & 83 & 16.6 \\
\hline Transcription error & 54 & 10.8 \\
\hline Dispensing error & 13 & 2.6 \\
\hline Administration error & 102 & 20.4 \\
\hline
\end{tabular}

*percentage has been calculated from total number of cases observed for each parameter (500).

\section{Prescribing error}


Out of five hundred prescriptions analysed, prescribing errors were observed in 83 prescriptions. A total of 110 errors were observed in those eighty three prescriptions. Thus $83.4 \%$ prescriptions were complete orders consisting of all parameters and without any prescribing error. The detailed distribution of different components of prescribing error is given in (Table 4). Fifty two prescriptions did not have complete general information i.e. patient name, date, registration number and signature of prescriber.

Table 4: Frequency and percentage of components of medication errors.

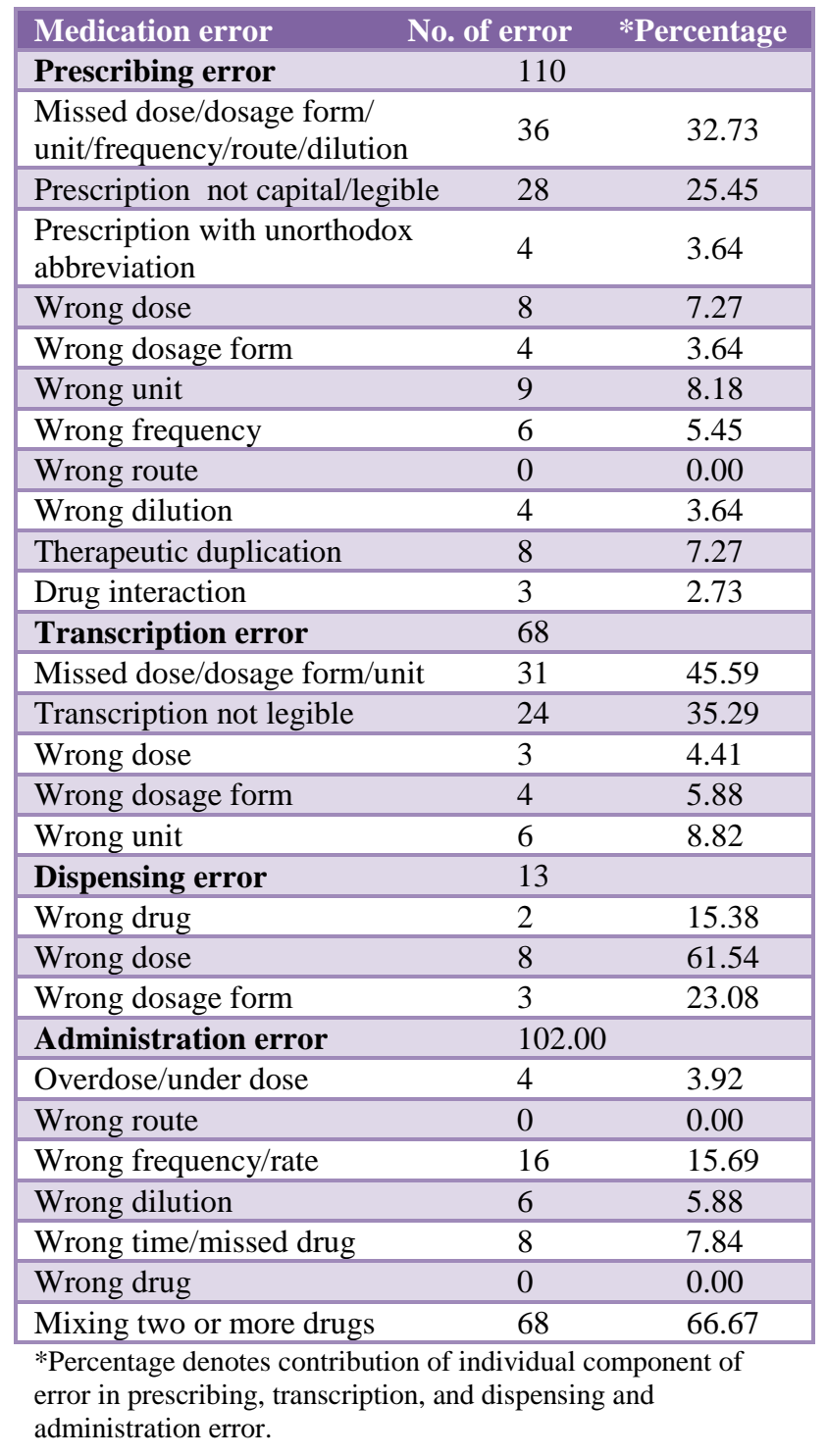

\section{Transcription error}

Out of five hundred prescriptions analysed, transcription errors were observed in 54 transcriptions. A total of 68 errors were observed in those 54 transcriptions. Thus $89.2 \%$ transcriptions were complete orders consisting of all parameters and without any transcribing error. The detailed distribution of different components of transcription errors is given in (Table 4). Forty six transcriptions did not have general information i.e. patient name, date, registration number and signature of prescriber.

\section{Dispensing error}

Out of the 500 dispensed events analysed, dispensing errors were observed in $13(2.6 \%)$ of the cases. The detailed distribution of different components of dispensing errors is given in (Table 4).

\section{Administration error}

Out of the 500 administration events analysed, administration errors were observed in 102 (20.4\%) of the cases. The detailed distribution of different components of administration errors is given in (Table 4).

\section{Severity of medication error}

The level of severity of medication error as proposed by NCC MERP has been given in (Table 5).

\section{Table 5: Categorization of errors as per NCC MERP} severity index.

\begin{tabular}{|lll|}
\hline Level of severity & Category & $\begin{array}{c}\text { percentage of } \\
\text { medication errors }\end{array}$ \\
\hline No error & Category A & 48.2 \\
\hline Error, no harm & Category B & 33.5 \\
\hline & Category C & 12.3 \\
\hline & Category D & 2.7 \\
\hline Error, harm & Category E & 2.3 \\
\hline & Category F & 1 \\
\hline & Category G & 0 \\
\hline & Category H & 0 \\
\hline Error, death & Category I & 0 \\
\hline
\end{tabular}

\section{DISCUSSION}

Unfortunately there is no standard method for calculation of medication error. Most commonly it is calculated as number of error divided by opportunities of error. ${ }^{22}$ Opportunities of medication error may be during prescribing, transcribing, administering and dispensing. Further there can be many opportunities of error in each of them. Arguably number of cases observed can be a valuable denominator in these circumstances. In these study five hundred cases of prescription and their subsequent transcription, dispensing and administration were observed and hence a denominator of five hundred has been chosen for calculating medication error rate. Out of five hundred cases, medication errors were identified in 293 cases. These included more than one error in the prescriptions and transcriptions. The total number of medication errors was $252(50.4 \%)$ if all error in a 
prescription or transcription were counted as one error. Since all forms of medication error has been taken from same patients we can say that on average $50 \%$ of patients received some form of medication error. These exclude errors committed in writing general information i.e. patient name, date, registration number and signature of prescriber. Classification of errors helps understand how it can be prevented.

\section{Prescribing error}

A prescribing error can result due to error in selecting drug, dose and duration appropriate to the patient indication or due to error in the act of writing prescription termed as prescription error. 23 In this study prescribing error was the most common cause of medication error $(37.54 \%)$. Appropriateness of the drug selection for indication was not checked as there is no institutional guidelines and lack of resources. Similarly though new MCI recommendations mandate writing of prescription in capital, the institute is still in the process of adopting these recommendations and it is not being followed uniformly. ${ }^{24}$ So we checked for either capital or legible prescription whichever was feasible. $25.45 \%$ of the prescribing errors were due to illegible prescriptions. Interestingly, frequency of error of omission (32.73\%) i.e. missed dose, dosage form, unit, frequency, route, dilution was almost similar to commission error of writing wrong dose, dosage form, unit, frequency, route, dilution and using unorthodox abbreviations (30.82\%). Eight cases of therapeutic duplications were found all of which were due to different physicians writing same class of drugs. General information such as date, patient initial, registration number and signature of the prescriber were missing in 52 prescriptions.

There is paucity of empirical studies pertaining to prescribing errors in India and they cannot be compared as the parameters assessed were different in different studies. A study done at rural tertiary care teaching hospital in Maharashtra, India on 499 prescriptions found general information about patient (name, age sex) were missing in $24 \%$ of prescriptions and signature of prescribers were missing in $12 \%$ of cases. Strength, duration and route of administration were missing from approximately $75 \%$ of prescriptions. ${ }^{25}$ Missing general information does not have direct impact on inpatient as they were written in files specific to the patient. Missing strength, duration and route are potentially dangerous as it leaves the matter to understanding of the nurses. Though it was found that in majority of the cases, nurses administered the drug correctly either with the help of senior nurses or by asking the prescribing physician. But it resulted into waste of time and can be source of potential harm. Illegible prescription is a potential error which can be easily avoided. We found two prescriptions which were unreadable due to many crosses, ticks and poor handwriting. In our study, around $25 \%$ of prescriptions were not legible which is very close $(17.6 \%)$ to above mentioned study. There are scores of examples of how illegible prescriptions led to use of wrong medicines and to disaster. ${ }^{26}$ Much has since been written about this problem but the problem still exists. Perhaps there are few things in medical culture which forces them to be legible. A notification by MCI to write the prescription in capital is a welcome step in this direction. But the notification still needs government approval. ${ }^{27}$

\section{Root cause analysis of prescribing error}

Inability to write (omission error) in most of the cases physicians thought that nurses knew the information since they are using these drugs regularly. Commission error was due to lack of knowledge, communication failure and errors in calculation of doses. As expected illegibility of prescription were said to be due to lack of time. Therapeutic duplication and drug interactions were due to inability of the physician to note the previous medications.

\section{Transcribing error}

Transcription errors consisted $23.21 \%$ of medication errors. A total of 68 transcription errors were observed in 54 transcriptions. Missed dose, dosage form, unit were most common causes of transcription error. Legibility of the transcription was also a problem as 24 transcripts were found illegible as compared to 28 prescriptions written by physicians. This is interesting as this aspect of the problem has never been highlighted. Wrong dose, dosage form and units were observed in 13 prescriptions (19\%). A similar study in teaching hospital in Tehran has found high rate of transcription error $(29.9 \%) .{ }^{28}$ Use of computerized provider order entry (CPOE) has reduced the error rate due to transcription compared to manual entry. In a study in USA, transcription related errors were 72.4 per month using a manual entry system of the orders, which was then reduced to 2.2 per month after implementing CPOE. ${ }^{29}$ In the hospitals using CPOE the prescription is directly presented to the pharmacy. But in most of the hospital prescription is copied to computer by a transcriptionist usually a pharmacist.

\section{Root cause analysis of transcription error}

Omission errors were due to failure to communicate with prescribing physician and commonness of the drug. A common practice was to copy the erroneous prescription as it is without understanding it. Lack of knowledge and inadequate training in junior staff were also found to be the causative factors.

\section{Dispensing error}

Dispensing error was checked by matching dispensed medicine against medication order sheet and prescription. Dispensing of different brand of same medicine was not considered as dispensing error. A total of 13 errors in dispensing were observed which was attributed to 
illegibility of medication order and communication failure.

\section{Administration error}

Administration errors were $2^{\text {nd }}$ most common cause of medication error observed in 102 cases (34.81\%). Administration error was checked by directly observing the drug preparation and administration process and checking nursing sheets. Use of wrong diluents or mixing incompatible drugs is preparation error. An error which has reached patient due to error in prescription or transcription has not been counted as administration error. Majority of the error were due to mixing of parenteral drug in same syringe. Mixing solutions of parenteral drugs is generally not recommended because of the potential for incompatibility and consequent loss of activity of one or both drugs. Decision to mix drugs was made without knowledge of their compatibility. Since there were no compelling reasons for giving two or more drugs in single syringe it has been considered irrational and an error. There were 68 instances when nurses mixed two or more drugs in a single syringe. A prospective observational study of 107 nurses preparing and administering 568 intravenous medications on six wards across two teaching hospitals in UK has found an administration error rate of $69.7 \%$. Four error types (wrong intravenous rate, mixture, volume, and drug incompatibility) accounted for majority (91.7\%) of these errors. $^{30}$ other studies utilizing direct observation for detection of administration error has reported an administration rate of around $18-27 \%$. $^{31,32}$

\section{Root cause analysis of administration error}

Mixing of two drugs is common practice in this hospital and the numbers might be higher if it is not being observed. It was found that most of the nurses were aware about this despite they did it as it was routine practice and convenient for them. Incompatibility problems are more likely to arise when small concentrated volumes are mixed in a syringe rather than in the larger volume of an infusion bag. This is because of higher mutual drug concentrations and potentially greater $\mathrm{pH}$ changes in the more concentrated solution. The absence of any visible change to a solution upon mixing does not automatically exclude degradation of either or both components.

Our study has several limitations. Due to small sample size the results can't be generalized. Further the results cannot be extrapolated to non-teaching hospitals. Despite our best efforts we might have failed to detect some errors particularly administration error as it has to be directly observed. Also, inappropriateness of the drug for the clinical indication has not been checked, which is detected most reliably using explicit criteria based on evidence rather than implicit criteria based on clinical judgment. Because nurses and physicians on the study wards were aware of the study, it may have affected both the occurrence and detection of errors. In addition, the incidence of errors could have been reduced as the study progressed because we were obliged to inform and correct serious practice problems.

\section{CONCLUSION}

This study addresses medication error involving physicians, nurses and pharmacist, to analyse their causes in tertiary care teaching hospital. It will help in taking decisions and formulating policies regarding preventive steps to avoid these medication errors intended to inpatient hospital setting.

From error to error one discovers the entire truth. - $\quad$ Sigmund Freud.

Funding: No funding sources

Conflict of interest: None declared

Ethical approval: The study was approved by the Institutional Ethics Committee

\section{REFERENCES}

1. Kopec D, Kabir MH, Reinharth D, Rothschild O, Castiglione JA. Human errors in medical practice: systematic classification and reduction with automated information systems. J Med Syst. 2003;27(4):297-313.

2. National coordinating council for medication error reporting and prevention (NCC MERP). What is a medication error? Available at http://www.nccmerp.org/about-medication-errors. Accessed 23 July 2015.

3. Linda KT, Corrigan JM, Donaldson MS. To err is human: building a safer health system. Washington, DC: The National Academies Press; 2000.

4. Bates DW, Spell N, Cullen DJ. The cost of adverse drug events in hospitalized patients. Adverse drug events prevention study group. JAMA. 1997;277:307-11.

5. Sewal RK, Singh PK, Prakash A, Kumar B, Medhi B. A prospective study to evaluate awareness about medication errors amongst health-care personnel representing North, East, West Regions of India. Int J Appl Basic Med Res. 2014;4(1):43-6.

6. Montesi G, Lechi A. Prevention of medication errors: detection and audit. British Journal of Clinical Pharmacology. 2009;67(6):651-5.

7. Dean B, Barber N. Validity and reliability of observational methods for studying medication administration errors. Am J Health Syst Pharm. 2001;58:54-9.

8. Morimoto T, Gandhi TK, Seger AC, Hsieh TC, Bates DW. Adverse drug events and medication errors: detection and classification methods. Qual Saf Health Care. 2004;13:306-14.

9. Drug and therapeutics committees - a practical guide, 2003. Available 
http://apps.who.int/medicinedocs/en/d/Js4882e/7.2.ht $\mathrm{ml}$.

10. American society of hospital pharmacists. ASHP guidelines on preventing medication error in Hospital. Am J Hosp Pharm. 1993;50;305-14.

11. Saghafi F, Zargarzadeh AH. Medication error detection in two major teaching hospitals: What are the types of errors? Journal of Research in Medical Sciences : The Official Journal of Isfahan University of Medical Sciences. 2014;19(7):617-23.

12. Franklin BD, Reynolds M, Shebl NA, Burnett S, Jacklin A. Prescribing errors in hospital inpatients: a three-centre study of their prevalence, types and causes. Postgrad Med J. 2011;87:739-45.

13. Hughes RG, Blegen MA. Medication administration safety. In: Hughes RG, editor. Patient safety and quality: an evidence-based handbook for nurses. Rockville (MD): Agency for Healthcare Research and Quality (US); 2008 April Chapter 37. Available at http://www.ncbi.nlm.nih.gov/books/NBK2656/.

14. NCC MERP Index for Categorizing Medication Errors, Available

at www.nccmerp.org/sites/default/files/taxonomy200107-31.pdf.

15. Drug Interactions Checker. Available at http://www.drugs.com/drug_interactions.php.

16. NCC MERP Taxonomy of Medication Errors available www.nccmerp.org/sites/default/files/taxonomy200107-31.pdf.

17. Wilson PF, Dell LD, Anderson GF. Root cause analysis: a tool for total quality management Milwaukee, Wisconsin: ASQ Quality Press; 1993:8-17.

18. Framework for conducting a root cause analysis and action plan. available at http://www.jointcommission.org/framework_for_con ducting_a_root_cause_analysis_and_action_plan/.

19. NCC MERP index for categorizing medication errors. available at http://www.nccmerp.org/typesmedication-errors.

20. Thakur H, Thawani V, Raina RS, Kothiyal G, Chakarabarty M. Noncompliance pattern due to medication errors at a teaching hospital in Srikot, India. Indian J Pharmacol. 2013;45:289-92.

21. Zeraatchi A, Talebian M, Nejati A, DashtiKhavidaki $\mathrm{S}$. Frequency and types of the medication errors in an academic emergency department in Iran: The emergent need for clinical pharmacy services in emergency departments. J Res Pharm Pract. 2013;2:118-22.

22. Donnell J, Cohen L, Iyer P, Medication errors and adverse drug reaction in hospitalized patient: how common are they and why do they occur? In: Donnell J, Ahuja GD. Drug Injury: Liability, Analysis, and Prevention. Lawyers and Judges Publishing Company; 2005:166.

23. Velo GP, Minuz P. Medication errors: prescribing faults and prescription errors. Br J Clin Pharmacol. 2009;67:624-8.

24. MCI prescription on legible writing hailed. Available at http://www.thehindu.com/news/cities/Kochi/mciprescription-on-legible-writinghailed/article5667413.ece.

25. Phalke VD, Phalke DB, Syed MMA, Mishra A, Sikchi S, Kalakoti P. Prescription writing practices in a rural tertiary care hospital in Western Maharashtra, India. The Australasian Medical Journal. 2011;4(1):4-8.

26. Bruner A, Kasdan ML. Handwriting errors: harmful, wasteful and preventable. J Ky Med Assoc. 2001;99(5):189-92.

27. Health ministry cure for doc's prescriptions, write in caps. Available at http://www.dailyexcelsior.com/health-ministry-curefor-docs-prescriptions-write-in-caps/.

28. Fahimi F, Abbasi Nazari M, Abrishami R, Sistanizad M, Mazidi T, Faghihi T, et al. Transcription errors observed in a teaching hospital. Arch Iran Med. 2009;12(2):173-5.

29. Barron WM, Reed RL, Forsythe S, Hecht D, Glen J, Murphy B, et al. Implementing computerized provider order entry with an existing clinical information system. Jt Comm J Qual Patient Saf. 2006;32:506-16.

30. Westbrook JI, Rob MI, Woods A, Parry D. Errors in the administration of intravenous medications in hospital and the role of correct procedures and nurse experience. BMJ quality and safety. 2011;20(12):1027-34.

31. Westbrook JI, Woods A, Rob MI, Dunsmuir WT, Day RO. Association of interruptions with increased risk and severity of medication administration errors. Arch Intern Med. 2010;170(8):683-90.

32. Barker KN, Flynn EA, Pepper GA, Bates DW, Mikeal RL. Medication errors observed in 36 healthcare facilities. Arch Intern Med. 2002;162(16):1897-903.

Cite this article as: Kumar S, Madhwar A, Pathak AK, Saikia D. Learning from error: identification and analysis of causative factors leading to medication error in an inpatient hospital setting. Int J Basic Clin Pharmacol 2016;5:999-1005. 